\title{
Life at the Bleeding Energy Edge: resource conservation strategies of microbes at Blood Falls, Antarctica
}

\author{
J.I. OHLSSON ${ }^{1 *}$, D. GHOSH ${ }^{1}$, R.A. VIRGINIA ${ }^{2}$, J.A. \\ MIKUCKI $^{1}$
}

${ }^{1}$ Dept. of Microbiology, University of Tennessee, Knoxville, TN 37996, USA (* correspondence: johlsson@utk.edu; jmikucki@utk.edu)

${ }^{2}$ Environmental Studies Program, Dartmouth College, Hanover, NH 03755, USA

(ross.a.virginia@dartmouth.edu)

\section{Subglacial Brine Microbiome}

A diverse microbiome has been found[1] inhabiting cold brine fluids below the Taylor Glacier in Taylor Valley, Antarctica, one of the McMurdo Dry Valleys. These fluids discharge episodically through Blood Falls at the tongue of the glacier into the west lobe of Lake Bonney, creating a striking display as the discharge is colored reddish brown by high concentrations of ferrous iron, sulfate, and salts.

The prokaryotic inhabitants of this brine must contend with these inorganic solutes. At the same time, they must tolerate low temperatures $\left(-7^{\circ} \mathrm{C}[2]\right)$ and survive with little or no new carbon input, as the thick glacier ice prevents photosynthesis. The possible sources of carbon and energy in this system are chemoautotrophy in interaction with surrounding rocks and brine, and chemoheterotrophy using relict organic matter from before the subglacial aquifer formed, perhaps some 1-2 million years ago[2].

In this study we have conducted bioinformatics analyses on a metagenome sequenced from Blood Falls subglacial brine in order to explore the metabolic adaptations and stress responses of microbes at this polyextreme site.

\section{Survey of Metabolic Capabilities}

Automatic annotation of the assembled metagenome in MG-RAST[3] reveals a varied array of capabilities. There is a significant presence of carbon fixation, including RuBisCo as well as the Wood-Ljungdahl pathway, a highly efficient single carbon fixation pathway that can conserve energy[4]. This and other features of the metagenome may represent adaptations to life in cold, dark, and saline environments, and may inform future investigations into the limits of life on Earth and other water-bearing worlds.

[1] Campen et al. (2019) Env Micro 21, 2290-2306. [2]

Mikucki et al. (2009) Science 324, 397-400. [3] Meyer et al. (2008) BMC Bioinf 9, 386. [4] Bar-Even et al. (2012) J Exp Bot 63, 2325-2342. 